\title{
Symmetric and asymmetric evaluation of the Tokamap in comparison with direct symplectic integration
}

\author{
Marc Eberhard* \\ Aston University, Birmingham, B4 7ET, United Kingdom \\ (Received 20 September 2004; published 24 February 2005)
}

\begin{abstract}
Comparing the Poincaré plots of the Tokamap and the underlying Hamiltonian system reveals large differences. This stems from the particular choice of evaluation of the singular perturbations present in the system (a series of $\delta$ functions). A symmetric evaluation approach is proposed and shown to yield results that almost perfectly match the Hamiltonian system.
\end{abstract}

DOI: 10.1103/PhysRevE.71.026411

PACS number(s): 52.65.Cc, 52.55.Dy, 05.45.Pq, 52.55.Fa

\section{INTRODUCTION}

Detailed transport studies in plasmas require the solution of the time evolution of many different initial positions of test particles in the phase space of the systems to be investigated [1]. To reduce the amount of numerical work, one would like to replace the time consuming integration of the time-continuous system with a fast discrete mapping [2]. Classic examples of this approach are the Chirikov-Taylor map [3] (also known as the standard map) and the Tokamap [4] proposed by Balescu et al.

These mappings are derived from kicked systems, such as the kicked rotator. This basically means that the system consists of an integrable part and a nonintegrable perturbation. The perturbation is only present at fixed singular points in time and zero for all other times. Thus the system remains integrable for all times except those when the perturbation is present. The assumption is, of course, that the physical and nonsingular perturbation of the true Hamiltonian system can be well approximated by the action of a "collapsed" perturbation in the form of a $\delta$ function.

The solution for these singular points is usually found by approaching the point from either later or earlier times $(t \pm \varepsilon)$ and calculating the limit for $\varepsilon \rightarrow 0$. It is clear that the exact values of the system variables at these singular points are mathematically not well defined. In a strict mathematical sense, there are only left-handed and right-handed limiting values, which are of course different. However, choosing one of the two limiting values does not necessarily yield the correct result [5] compared to a full integration of the underlying Hamiltonian system. This can be seen by comparing the results from a map with the numerical calculation of the given system with an approximative $\delta$ function. This will be shown in this paper for the example of the Tokamap after reviewing its derivation.

A symmetric procedure to evaluate the system at these singular points is then presented and the near perfect agreement with the result of the numerical calculation is demonstrated. The $\delta$ functions in the mapping are approximated with steep Gaussian functions for the numerical integration

\footnotetext{
*Electronic address: m.a.eberhard@aston.ac.uk; URL: http:// www.aston.ac.uk/ $/$ eberhama/
}

and a symplectic integrator is used to assure a high level of accuracy even for chaotic regions of the phase space.

\section{THE TOKAMAP HAMILTONIAN}

For the derivation of the Tokamap we start from the Hamiltonian

$$
H=\int^{\psi} d \psi^{\prime} W\left(\psi^{\prime}\right)-\frac{K}{(2 \pi)^{2}} \frac{A \psi}{1+A \psi} \cos (2 \pi \theta) f(t),
$$

which describes the motion of magnetic field lines in a Tokamak. It is quite often used as a simple approximation for the real magnetic field found in the experiments. It describes a system with a given winding number $W(\psi)$ (integrable part) and a perturbation (nonlinear and nonintegrable) that will vanish for $\psi=0$. This specific form of the perturbation was introduced by Balescu et al. [4] to avoid the unphysical case with $\psi<0$, which occurs in the standard map.

Thus we obtain the equations of motion for the field lines

$$
\begin{gathered}
\dot{\psi}=-\frac{\partial H}{\partial \theta}=-\frac{K}{2 \pi} \frac{A \psi}{1+A \psi} \sin (2 \pi \theta) f(t), \\
\dot{\theta}=\frac{\partial H}{\partial \psi}=W(\psi)-\frac{K}{(2 \pi)^{2}} \frac{A}{(1+A \psi)^{2}} \cos (2 \pi \theta) f(t),
\end{gathered}
$$

and choose the winding number

$$
W(\psi)=\frac{1}{1+d \psi} \quad \text { with } \quad d=3
$$

to be a realistic approximation for the magnetic field configuration in the TEXTOR-94 experiment [6]. Other choices are of course possible to model different experimental setups.

\section{DERIVATION OF THE TOKAMAP}

Since the generic case with a smooth and distributed function $f(t)$ cannot be integrated analytically, we have to choose this function as a sum of $\delta$ functions for the derivation of the Tokamap 


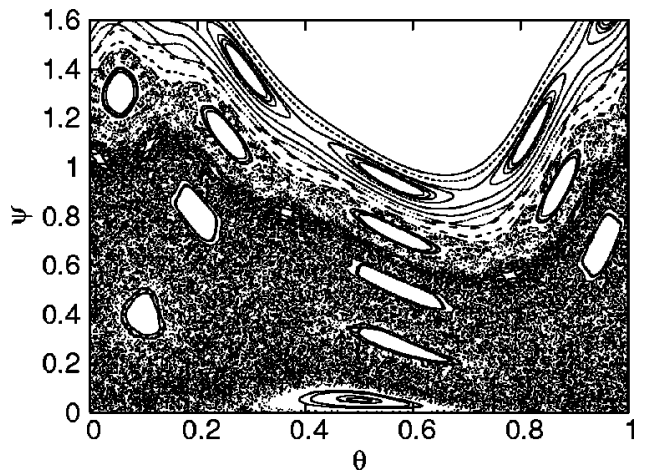

FIG. 1. Poincaré plot of the Tokamap for $A=1$ and $K=6$ as a phase space portrait.

$$
f(t)=\sum_{k=-\infty}^{\infty} \delta(k-t)
$$

and as a sum of steep Gaussian functions for the numerical integration as will be shown later. By using the left-handed limiting value of the $\delta$ functions we obtain a mapping for our Hamiltonian system

$$
\begin{gathered}
\psi_{n+1}=\psi_{n}-\frac{K}{2 \pi} \frac{A \psi_{n}}{1+A \psi_{n}} \sin \left(2 \pi \theta_{n}\right), \\
\theta_{n+1}=\theta_{n}+W\left(\psi_{n+1}\right)-\frac{K}{(2 \pi)^{2}} \frac{A}{\left(1+A \psi_{n}\right)^{2}} \cos \left(2 \pi \theta_{n}\right),
\end{gathered}
$$

which is almost identical to the Tokamap proposed by Balescu et al. [4],

$$
\begin{gathered}
\psi_{n+1}=\psi_{n}-\frac{K}{2 \pi} \frac{A \psi_{n+1}}{1+A \psi_{n+1}} \sin \left(2 \pi \theta_{n}\right), \\
\theta_{n+1}=\theta_{n}+W\left(\psi_{n+1}\right)-\frac{K}{(2 \pi)^{2}} \frac{A}{\left(1+A \psi_{n+1}\right)^{2}} \cos \left(2 \pi \theta_{n}\right),
\end{gathered}
$$

with the notable exception of the index of $\psi$ in the perturbation terms on the right-hand side of the equations. While we obtained $n$ in our analytical integration of the system, the Tokamap contains $n+1$. The reason is that Balescu et al. derived the Tokamap from the canonical transformation

$$
F\left(\psi_{n+1}, \theta_{n}\right)=\psi_{n+1} \theta_{n}+F_{0}\left(\psi_{n+1}\right)+K \delta F\left(\psi_{n+1}, \theta_{n}\right)
$$

with

$$
F_{0}(\psi)=\int^{\psi} d \psi^{\prime} W\left(\psi^{\prime}\right)
$$

and

$$
\delta F\left(\psi_{n+1}, \theta_{n}\right)=-\frac{1}{(2 \pi)^{2}} \frac{A \psi_{n+1}}{1+A \psi_{n+1}} \cos \left(2 \pi \theta_{n}\right)
$$

as the generating function instead of integrating the equations of motion. The advantage of their method is that the resulting mapping is guaranteed to be symplectic. However, it must be noted that Eq. (1) is-strictly speaking-only the Hamiltonian for the Tokamap for a vanishing perturbation

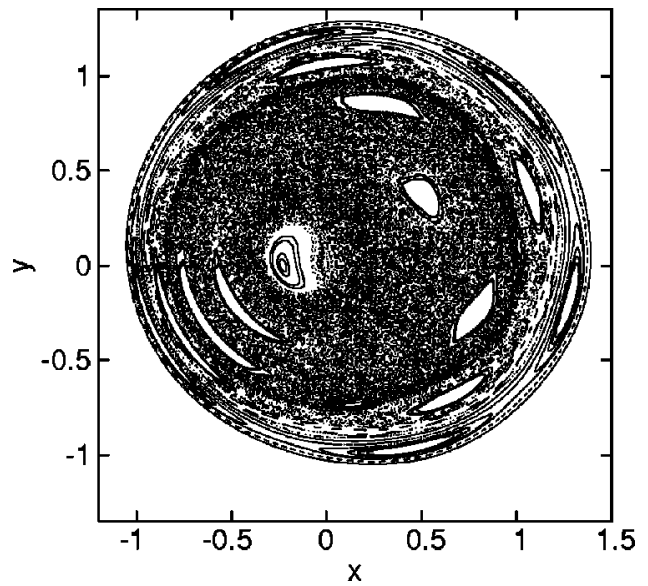

FIG. 2. Poincare plot of the Tokamap for $A=1$ and $K=6$ as a polar plane for a plasma with a circular cross section.

term [4]. Nevertheless, as the perturbation is assumed to be small compared to the integrable part of the Hamiltonian system, it is commonly assumed that the mapping derived through a canonical transformation still closely corresponds to the original Hamiltonian system in question.

The Poincaré plots of this mapping are shown for a perturbation strength of $K=6$ both in Hamiltonian coordinates (phase space) in Fig. 1 and in real toroidal geometry in Fig. 2 for a plasma with a circular cross section as is the case for TEXTOR-94 [6].

One can clearly see the typical structure of such a system with some still intact Kolmogorov-Arnol'd-Moser (KAM) tori and chaotic regions in between. For transport studies it is extremely important that the chaotic regions are at the correct positions in the phase space and that the correct KAM tori are still intact, when compared to the underlying Hamiltonian system. The usability of a mapping entirely depends on its ability to reproduce these features correctly.

\section{FULL NUMERICAL INTEGRATION OF THE TOKAMAP HAMILTONIAN}

To assess the quality of the mapping, we compare the results from the Tokamap with the results from a full numerical integration of the Hamiltonian [2] with a symplectic integrator $[7,8]$. The sum over the $\delta$ functions is approximated by an integration over a sum of steep Gaussian functions

$$
f(t, a)=\sum_{k=-\infty}^{\infty} \frac{a}{\sqrt{\pi}} \exp \left\{-a^{2}(t-k)^{2}\right\}
$$

and the results in Figs. 3 and 4 do show very disappointing differences. Not only are the positions of the islands and KAM tori wrong, but even worse, there are far more broken KAM tori in the Tokamap than in the numerical integration.

\section{SYMMETRIC MAP}

Further investigation reveals quickly that the differences are caused by the way the $\delta$ functions are evaluated. In particular the restriction to use only the left- or right-handed 


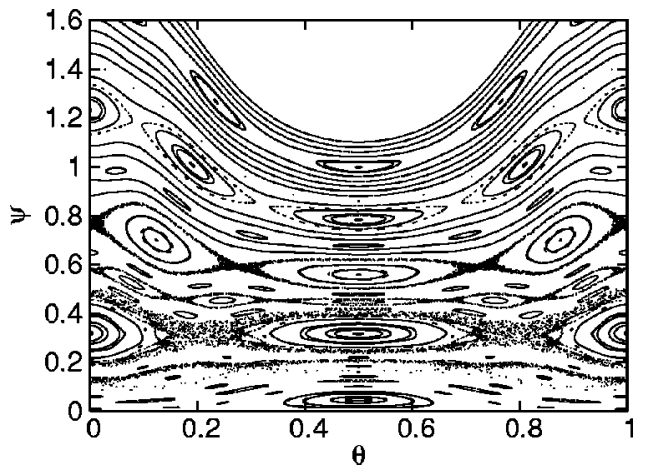

FIG. 3. Poincaré plot for a symplectic integration with $A=1$, $K=6, \Delta t=10^{-3}$, and $a=200$ as a phase space portrait.

limiting value destroys the symmetry of the solution seen from the results of the integration.

However, this symmetry can be restored if the $\delta$ functions are evaluated as shown in Fig. 5. We interpret the $\delta$ function as the limiting case of a nonsingular perturbation, which is symmetrically present around the location of the $\delta$ functions. It is thus the limiting case of our numerical integration in the limit $a \rightarrow \infty$.

In a physical sense we apply half of the $\delta$ function before the actual time step and half of it afterward. It is important to stress that this particular choice is mathematically as arbitrary as any other value between the left- and right-handed limiting values. However, looking at the system from a physical point of view and interpreting the $\delta$ function as a "collapsed" perturbation makes this a very plausible choice.

Starting from Eq. (1) with

$$
f(\psi)=-\frac{K}{(2 \pi)^{2}} \frac{A \psi}{1+A \psi}
$$

and

$$
g(\theta)=\cos (2 \pi \theta)
$$

leads to the equations of motion for the system

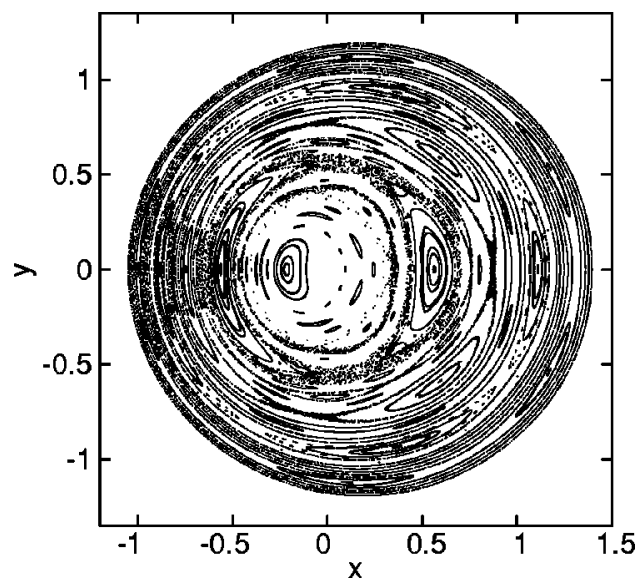

FIG. 4. Poincaré plot for a symplectic integration with $A=1$, $K=6, \Delta t=10^{-3}$, and $a=200$ as a polar plane for a plasma with a circular cross section.

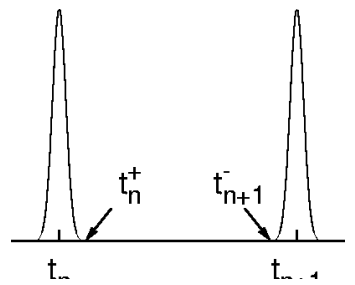

FIG. 5. Notation used for the construction of the symmetric Tokamap.

$$
\begin{gathered}
\dot{\psi}=-\frac{\partial H}{\partial \theta}=-f(\psi) \frac{\partial g(\theta)}{\partial \theta} \sum_{k=-\infty}^{\infty} \delta(t-k), \\
\dot{\theta}=\frac{\partial H}{\partial \psi}=W(\psi)+\frac{\partial f(\psi)}{\partial \psi} g(\theta) \sum_{k=-\infty}^{\infty} \delta(t-k),
\end{gathered}
$$

and we can now evaluate this for the three steps from $t_{n}$ to $t_{n}^{+}$ to $t_{n+1}^{-}$and finally to $t_{n+1}$.

The first step from $t_{n}$ to $t_{n}^{+}$includes half of the $\delta$ function at $t_{n}$,

$$
\begin{aligned}
& \psi_{n}^{+}=\psi_{n}-\frac{1}{2} f\left(\psi_{n}\right) \frac{\partial g\left(\theta_{n}\right)}{\partial \theta}, \\
& \theta_{n}^{+}=\theta_{n}+\frac{1}{2} \frac{\partial f\left(\psi_{n}\right)}{\partial \psi} g\left(\theta_{n}\right),
\end{aligned}
$$

and then we continue through the integrable part of the system from $t_{n}^{+}$to $t_{n+1}^{-}$,

$$
\psi_{n+1}^{-}=\psi_{n}^{+}, \quad \theta_{n+1}^{-}=\theta_{n}^{+}+W\left(\psi_{n}^{+}\right),
$$

and finally include the first half of the $\delta$ function at $t_{n+1}$ from $t_{n+1}^{-}$to $t_{n+1}$,

$$
\begin{aligned}
& \psi_{n+1}=\psi_{n+1}^{-}-\frac{1}{2} f\left(\psi_{n+1}\right) \frac{\partial g\left(\theta_{n+1}\right)}{\partial \theta}, \\
& \theta_{n+1}=\theta_{n+1}^{-}+\frac{1}{2} \frac{\partial f\left(\psi_{n+1}\right)}{\partial \psi} g\left(\theta_{n+1}\right) .
\end{aligned}
$$

Eliminating the intermediate stages $t_{n}^{+}$and $t_{n+1}^{-}$leads to the final form of the symmetric Tokamap:

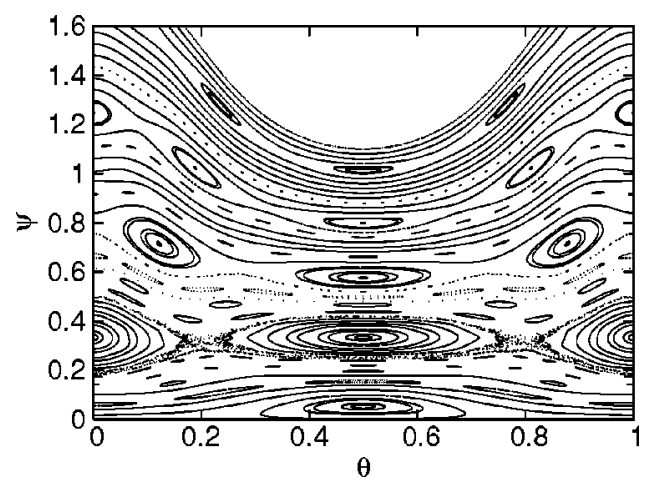

FIG. 6. Poincaré plot of the symmetric Tokamap for $A=1$ and $K=6$ as a phase space portrait. 


$$
\begin{gathered}
\psi_{n+1}=\psi_{n}-\frac{1}{2} f\left(\psi_{n}\right) \frac{\partial g\left(\theta_{n}\right)}{\partial \theta}-\frac{1}{2} f\left(\psi_{n+1}\right) \frac{\partial g\left(\theta_{n+1}\right)}{\partial \theta}, \\
\theta_{n+1}=\theta_{n}+W\left(\psi_{n}-\frac{1}{2} f\left(\psi_{n}\right) \frac{\partial g\left(\theta_{n}\right)}{\partial \theta}\right)+\frac{1}{2} \frac{\partial f\left(\psi_{n}\right)}{\partial \psi} g\left(\theta_{n}\right) \\
+\frac{1}{2} \frac{\partial f\left(\psi_{n+1}\right)}{\partial \psi} g\left(\theta_{n+1}\right) .
\end{gathered}
$$

The resulting map is implicit and must be iterated for evaluation. The results are shown in Figs. 6 and 7. They show a very good agreement with Figs. 3 and 4, which justifies the physical interpretation of the $\delta$ functions over a more stringent mathematical choice. Instead of using a simple physical interpretation of the perturbation term, it is also possible to use a more formal approach based on canonical transforms [9]. However, that approach is far more complex and not as simple and elegant as this one.

\section{CONCLUSIONS}

The comparison of the Tokamap with the numerical integration of the true Hamiltonian system shows significant differences and leads to the proposal of a symmetric Tokamap based on a different evaluation of the $\delta$ functions. The more

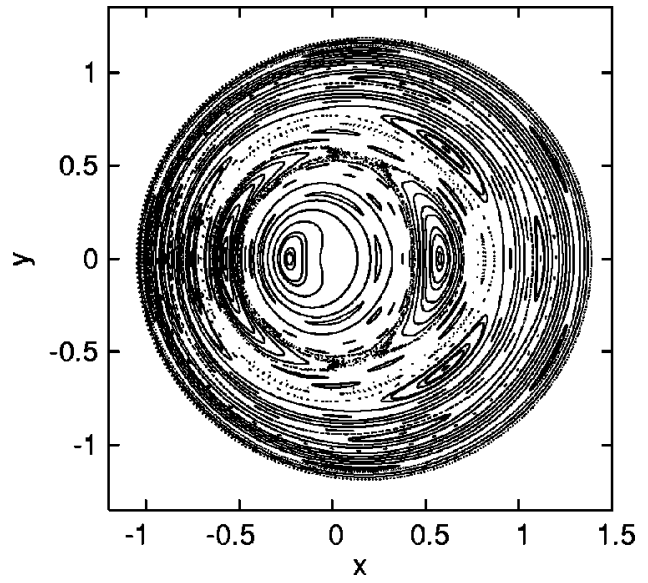

FIG. 7. Poincaré plot of the symmetric Tokamap for $A=1$ and $K=6$ as a polar plane for a plasma with a circular cross section.

physically motivated approach is in very good agreement with the numerical integration, while the more "mathematical correct" derivation of the Tokamap yields a quite different picture. This important result must be taken into account, when constructing a map for a given Hamiltonian system to reduce computational time in transport studies.
[1] K. H. Spatschek, M. Eberhard, and H. Friedel, Phys. Mag. 20, 85 (1998)

[2] M. Eberhard, Ph.D. thesis, Heinrich-Heine-Universität, Düsseldorf, 2000 (unpublished).

[3] B. V. Chirikov, Phys. Rep. 52, 263 (1979).

[4] R. Balescu, M. Vlad, and F. Spineanu, Phys. Rev. E 58, 951 (1998).

[5] M. Eberhard, Europhysics Conference Abstracts: EPS Conference on Controlled Fusion and Plasma Physics (European
Physical Society, Maastricht, The Netherlands, 1999), Vol. 23J, pp. 781-784.

[6] K. H. Finken, Technical Report, Institut für Plasmaphysik, Forschungszentrum Jülich GmbH, Association EURATOM-KFA, Germany, 1996 (unpublished).

[7] E. Forest and R. D. Ruth, Physica D 43, 105 (1990).

[8] H. Yoshida, Phys. Lett. A 150, 262 (1990).

[9] S. S. Abdullaev, Nucl. Fusion 44, S12 (2004). 